\title{
UMA MISTURA DE FUNÇÕES BARREIRA E BARREIRA MODIFICADA NA RESOLUÇÃO DO FLUXO DE POTÊNCIA ÓTIMO
}

\author{
Jéssica Antonio Delgado \\ Prog. de Pós-Grad. Eng. Elétrica - FEB - Unesp - Av. Eng. Luiz E. C. Coube, 14-01. Bauru-SP \\ jessica_dellgado@hotmail.com \\ Edméa Cássia Baptista \\ Depto. de Matemática - FC - Unesp - Av. Eng. Luiz E. C. Coube, 14-01. Bauru-SP \\ baptista@fc.unesp.br \\ Edilaine Martins Soler \\ Depto. de Matemática - FC - Unesp - Av. Eng. Luiz E. C. Coube, 14-01. Bauru-SP \\ edilaine@fc.unesp.br \\ Antonio Roberto Balbo \\ Depto. de Matemática - FC - Unesp - Av. Eng. Luiz E. C. Coube, 14-01. Bauru-SP \\ arbalbo@fc.unesp.br \\ Leonardo Nepomuceno \\ Depto. de Engenharia Elétrica - FEB - Av. Eng. Luiz E. C. Coube, 14-01. Bauru-SP \\ leo@feb.unesp.br
}

\begin{abstract}
Resumo Uma abordagem misturada da função barreira e a função barreira modificada é investigada neste trabalho. As restrições de desigualdade são transformadas em igualdades introduzindo variáveis de folga positivas, as quais são tratadas pelas funções Barreira Logarítmica ou Barreira Modificada Logarítmica e as demais restrições através de multiplicadores de Lagrange. Estes métodos serão utilizados em duas etapas. Inicialmente aplicamos o método baseado na função Barreira Logarítmica até que uma condição de parada seja satisfeita, depois aplicamos o método baseado na função Barreira Modificada Logarítmica até que a condição de convergência seja satisfeita. Testes numéricos utilizando o sistema elétrico teste de 3 barras indicam que o método é eficiente na resolução do problema Fluxo de Potência Ótimo.
\end{abstract}

Palavras-chave: Fluxo de Potência Ótimo, Otimização não-linear, método de Newton, função Barreira, Função Barreira Modificada.

Abstract An approach mixed the barrier function and modified barrier function is investigated in this paper. The inequality constraints are transformed into equalities by adding non-negative slack variables, which are handled by logarithmic barrier function or logarithmic modified barrier function, while the remaining constraints are handled by means of the Lagrange multipliers method. These methods will be used in two steps. Initially we apply the logarithmic barrier function until a stop condition is satisfied, and then we apply logarithmic modified barrier function until a convergence condition is satisfied. Numerical tests carried out with the 3 bus electrical system indicate that the proposed approach is efficient in the resolution the optimal power flow problem.

Keywords: Optimal Power Flow, nonlinear optimization, Newton method, barrier function, modified barrier function. 


\section{INTRODUÇÃO}

Vários métodos de Otimização foram desenvolvidos após o surgimento do computador. A proposição, desenvolvimento e aplicação de um determinado método de Otimização a um problema real depende, basicamente, das características do problema, isto é, o problema ser linear, não-linear, convexo, inteiro, dinâmico, entre outras. Os métodos mistos têm sido amplamente investigados e utilizados principalmente na resolução de problemas de Otimização Não Linear, com bom desempenho, em problemas de grande porte. O objetivo da mistura dos métodos é aproveitar as melhores características de cada um e tentar driblar suas dificuldades.

O problema de Fluxo de Potência Ótimo foi proposto por Carpentier, no início da década de 60, a partir do problema de Despacho Econômico. Ele é representado matematicamente por um problema de Otimização Não Linear, com restrições de igualdade e desigualdade, não-convexo, de grande porte com variáveis contínuas e/ou inteiras, o qual determina o melhor ponto de operação do sistema, satisfazendo algum objetivo. Ele resolve o problema de Fluxo de Potência, uma vez que as restrições de igualdades são as equações do Fluxo de Potência e, ainda, otimiza uma função objetivo, a qual representa alguma característica do desempenho elétrico do sistema, satisfazendo as restrições de operação. Um caso particular deste problema é o FPO Reativo, no qual os controles ativos são fixados e as variáveis de controle relacionadas com a potência reativa como: as magnitudes da tensão dos geradores e dos compensadores síncronos e estáticos de potência reativa e os taps dos transformadores são otimizadas em relação à função objetivo.

Muitos trabalhos foram desenvolvidos na tentativa de resolvê-lo utilizando diferentes técnicas de Otimização Não-Linear, Linear e Métodos Heurísticos, entre eles citam-se: GRANVILLE [12], WU et al. [22] e TORRES \& QUINTANA [21] os quais aplicaram um algoritmo Primal-Dual Barreira Logarítmica na resolução do problema de Fluxo de Potência Ótimo e, as condições de Karush-Kuhn-Tuker (KKT) foram satisfeitas utilizando o método de Newton, sendo a maior dificuldade do algoritmo a escolha do parâmetro de Barreira; BAPTISTA et al. [1-3] resolveram o problema utilizando uma função Lagrangiana Aumentada-Barreira Logarítmica; BAPTISTA et al.[4] e SOUSA et al. [18-20] utilizaram uma função Lagrangiana Barreira Modificada. Mais recentemente, lembra-se de CAPITANESCU et al. [5-7] que analisa o desempenho de três algoritmos de pontos interiores e sua aplicação ao FPO.

Assim, tendo em vista a relevância do problema de FPO e a necessidade de se buscar métodos mais eficientes para a sua resolução, propõe-se, neste trabalho, uma solução para o problema de FPO por meio de uma abordagem que mistura a função barreira logarítmica e função barreira modificada logarítmica.

O Método da Função Barreira, ou método de Barreira, é utilizado para a resolução de problemas com restrições de desigualdade, cujo interior é não vazio. A função Barreira logarítmica foi estudada por FRISCH [11] para problemas de programação convexa. Outra função barreira, denominada função barreira inversa foi proposta por CARROL [8]. O método de barreira foi realmente popularizado por FIACCO\&McCORMICK [10], que realizaram um estudo teórico mais detalhado do método e desenvolveram um novo; associando a função barreira e a função penalidade em uma mesma função auxiliar.

O método da função Barreira Modificada foi desenvolvida por Polyak em 1992. Estes métodos combinam as melhores propriedades da função Lagrangiana Clássica e da função Barreira Clássica, evitando os problemas que ambas enfrentam. O método da função Barreira Modificada transforma o problema restrito em um outro problema equivalente, o qual é 
irrestrito e resolve uma sequência de problemas irrestritos até atingir a solução ótima. Estas funções são definidas através da relaxação do conjunto de restrições factíveis. Este método tem as seguintes vantagens: atinge o ótimo mesmo que a solução ótima esteja na fronteira da região factível do problema original, o parâmetro de barreira não precisa tender a zero e o condicionamento da matriz Hessiana é melhorado se a sequência de pontos tender para uma solução próxima à fronteira do problema.

A abordagem misturada, investigada neste trabalho, e aplicada na resolução do problema de Fluxo de Potência Ótimo é baseada no trabalho de GRIVA [13] Nesta, as restrições de desigualdade do problema são transformadas em igualdade através da utilização de variáveis de folga e excesso positivas. As variáveis de folga e excesso são tratadas pelas funções Barreira ou Barreira Modificada e as demais restrições através de multiplicadores de Lagrange. Os métodos serão utilizados em duas etapas. Inicialmente aplicamos o método baseado na função Barreira Logarítmica até que uma condição de parada seja satisfeita, depois aplicamos o método baseado na função Barreira Modificada Logarítmica até que uma condição de convergência seja satisfeita.

Testes com o sistema elétrico de 3 barras foram realizados para verificar a eficiência da abordagem proposta.

Este trabalho está organizado da seguinte forma: na seção 2 descreve-se o problema de FPO Reativo, na seção 3 apresenta-se o método misto da Função Barreira Logarítmica e da Função Barreira Modificada Logarítmica, na seção 4 apresentam-se os testes e resultados e finalmente, na seção 5, as conclusões do trabalho são discutidas.

\section{O PROBLEMA DE FPO REATIVO}

O problema de FPO Reativo com função objetivo perdas ativas na transmissão é representado matematicamente por:

$$
\begin{aligned}
& \text { Minimizar } f(x) \\
& \text { sujeito a } \mathrm{g}(x)=0 \\
& h(x) \leq 0 \\
& x_{\min } \leq x \leq x_{\max }
\end{aligned}
$$

em que $x^{T}=(v, \theta, t) \in R^{n}$ : vetor das variáveis de estado e de controle; $v$ : tensão elétrica; $\theta$ : ângulo de defasagem; t: tap dos transformadores. $f(x)$ : função objetivo que representa as perdas de potência ativa na transmissão; $g(x)=0 \in R^{m}$ : vetor que corresponde as equações do fluxo de potência; $h(x) \leq 0 \in R^{p}$ : vetor que corresponde restrições funcionais, que representam as gerações de potência reativa nas barras de controle de reativo; $x_{\text {min }} ; x_{\text {max }}$ : limites inferiores e superiores das variáveis.

\section{O MÉTODO MISTO DA FUNÇÃO BARREIRA E BARREIRA MODIFICADA}

\subsection{O MÉTODO DA BARREIRA LOGARÍTMICA}

Ao problema (1), segundo SOUSA [16], acrescentam-se variáveis de folga positivas para transformar as restrições de desigualdade em restrições de igualdade. As variáveis de 
folgas positivas são tratadas através da Função Barreira Logarítmica e as restrições através dos multiplicadores de Lagrange.

Desta forma define-se a seguinte função Lagrangiana Barreira Logarítmica:

$$
\begin{aligned}
L\left(x, \lambda, \pi_{1}, \pi_{2}, \pi_{3}, \mathrm{~s}_{1}, \mathrm{~s}_{2}, \mathrm{~s}_{3}\right)=f(x)-\mu \ln s_{1}-\mu \ln s_{2}-\mu \ln s_{3}+\lambda^{T} g(x) \\
+\pi_{1}\left(h(x)-s_{1}\right)+\pi_{2}\left(x+s_{2}-x_{\max }\right)+\pi_{3}\left(x-s_{3}-x_{\min }\right)
\end{aligned}
$$

em que $\lambda \in R^{m}, \pi_{1} \in R^{p}$, $\pi_{2}$ e $\pi_{3} \in R^{n}$ são os vetores dos multiplicadores de Lagrange, $\mu$ é o parâmetro de barreira e $s_{i}, i=1, \ldots, 3$ são as variáveis de folga. Neste método, uma sequência de problemas irrestritos, cuja função objetivo é dada por (2) será resolvida, para $\mu$ fixo, através do método de Newton.

\subsubsection{O MÉTODO DA BARREIRA MODIFICADA LOGARÍTMICA}

Ao problema (1), segundo o trabalho de SOUSA [17], acrescentam-se variáveis de folga positivas para transformar as restrições de desigualdade em restrições de igualdade. $\mathrm{Na}$ sequência, aplica-se uma relaxação, nas condições de não negatividade do problema, isto é nas variáveis de folga, utilizando o parâmetro de barreira. Essa relaxação resulta na expansão da região factível do problema original.

A Função Barreira Modificada Logarítmica proposta por POLYAK [15] é utilizada para tratar as variáveis de folga e às restrições de igualdade são associadas multiplicadores de Lagrange. Desta forma, define-se a seguinte função Lagrangiana Barreira Modificada Logarítmica:

$$
\begin{gathered}
L\left(x, \lambda, \pi_{1}, \pi_{2}, \pi_{3}, \mathrm{~s}_{1}, \mathrm{~s}_{2}, \mathrm{~s}_{3}\right)=f(x)-\mu\left(\pi_{1} \ln \left(\mu^{-1} s_{1}+1\right)\right)-\mu\left(\pi_{2} \ln \left(\mu^{-1} s_{2}+1\right)\right)-\mu\left(\pi_{3} \ln \left(\mu^{-1} s_{3}+1\right)\right) \\
+\lambda^{T} g(x)+\pi_{1}\left(h(x)-s_{1}\right)+\pi_{2}\left(x+s_{2}-x_{\max }\right)+\pi_{3}\left(x-s_{3}-x_{\min }\right)
\end{gathered}
$$

sendo $\lambda \in R^{m}, \pi_{1} \in R^{p}, \pi_{2}$ e $\pi_{3} \in R^{n}$ os vetores dos multiplicadores de Lagrange, $\mu$ é o parâmetro de barreira e $s_{i}, i=1, \ldots, 3$ são as variáveis de folga. Uma sequência de problemas irrestritos será resolvida, utilizando (3) para $\mu$ fixo e o método de Newton.

Lembra-se que para os dois métodos utilizam-se os passos primais e duais. Adotou-se para o cálculo do passo máximo uma adaptação da estratégia utilizada por GRANVILLE [12], TORRES e QUINTANA [21], entre outros. O passo primal é determinado pelo menor valor entre os componentes das variáveis de folga positivas e o valor 1 . O objetivo do passo primal é garantir a positividade das variáveis de folgas na solução e é dado por:

$$
\alpha_{p}=\sigma \min \left\{\left(\min _{\Delta S_{1}<0 \mathrm{e} \mathrm{S}_{1}>0} \frac{-S_{1}}{\Delta s_{1}}, \min _{\Delta S_{2}<0 \mathrm{eS}_{2}>0} \frac{-S_{2}}{\Delta s_{2}}, \min _{\Delta S_{3}<0 \mathrm{e}_{3}>0} \frac{-S_{3}}{\Delta s_{3}}, 1\right)\right\}
$$

O passo dual é calculado de forma que cada componente dos vetores duais $\lambda, \pi_{1}, \pi_{2}$ e $\pi_{3}$ permaneçam com seus respectivos sinais, isto é:

$$
\alpha_{d}=\sigma \min \left\{\left(\min _{\Delta \pi_{1}<0 \mathrm{e} \pi_{1}>0} \frac{-\pi_{1}}{\Delta \pi_{1}}, \min _{\Delta \pi_{2}>0 \mathrm{e} \pi_{2}<0} \frac{\pi_{2}}{\Delta \pi_{2}}, \min _{\Delta \pi_{3}<0 \mathrm{e} \pi_{3}>0} \frac{-\pi_{3}}{\Delta \pi_{3}}, 1\right)\right\}
$$


em que $\sigma$ é adotado igual a 0,9995 .

A atualização do parâmetro de Barreira $\mu$ para o método da Função Barreira e da Função Barreira Modificada é dada por:

$$
\mu_{k+1}=\frac{\mu_{k}}{\beta}
$$

em que $\beta$ é adotado igual a 10 .

\subsubsection{A MISTURA DAS FUNÇÕES BARREIRA E BARREIRA MODIFICADA}

Propõe-se, baseado no trabalho de GRIVA[13] utilizar o Método da Barreira Logarítmica e o Método da Barreira Modificada Logarítmica de uma maneira mista na resolução de um problema de FPO.

A ideia consiste em: aplicar o método da Barreira Logarítmica até que um critério de parada seja satisfeito e em seguida aplicar o método da Barreira Modificada Logarítmica. Neste trabalho, adota-se como critério de parada, para o Método de Barreira Logarítmica, um número máximo de iterações, para inicialmente verificar a aplicabilidade do método ao problema de FPO.

O critério de parada adotado para o Método da Barreira Modificada, sendo um teste para verificar a convergência do algoritmo, é adotado como apresentado por TORRES e QUINTANA [21] da seguinte forma:

$$
\begin{aligned}
& v_{1}^{k} \leq \xi_{1}, \quad \text { sendo, } \\
& v_{1}=\left\|g\left(x^{k}\right)\right\|_{\infty} \\
& v_{2}^{k} \leq \xi_{2} \\
& v_{3}^{k} \leq \xi_{3} \\
& v_{2}=\frac{\left|f\left(x^{k}\right)-f\left(x^{k-1}\right)\right|}{1+\left|f\left(x^{k}\right)\right|} \\
& v_{3}=\max \left\{\mathrm{h}_{j}(x)\right\}, j=1, \ldots, r
\end{aligned}
$$

\section{TESTES E RESULTADOS}

A aplicação da abordagem proposta, ao problema de FPO Reativo será detalhada num sistema exemplo de 3 barras para verificar a eficiência do método proposto.

\subsection{EXEMPLO ILUSTRATIVO}

O sistema exemplo de 3 barras apresentado por DOMMEL e TINNEY [9], será utilizado para detalhar a abordagem proposta. A Fig. 1 apresenta os dados do sistema.

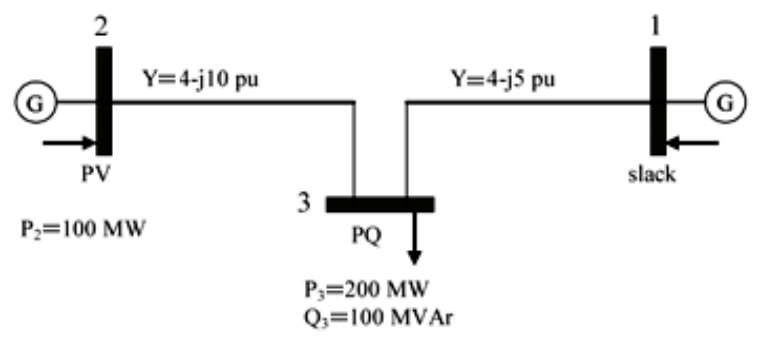

Figura 1: Sistema de 3 barras. 
Em que: a barra 1, slack, é a barra de referência angular; a barra 2, é a barra de geração e a barra 3, é a barra de carga. O objetivo do método é minimizar a função objetivo que representa as perdas de potência ativa na transmissão, atendendo as restrições de igualdade de potência ativa e reativa, com duas restrições de desigualdade canalizadas, as quais representam o controle de reativo dos geradores (barras 2) e variáveis canalizadas que representam as tensões nas barras (barras 1, 2 e 3 ).

O problema pode ser representado como:

$$
\begin{aligned}
& \text { Minimizar } f(v, \theta) \\
& \text { sujeito a } \Delta \mathrm{P}_{2}=0 \\
& \Delta P_{3}=0 \\
& \Delta \mathrm{Q}_{3}=0 \\
& \underset{\min 2}{\mathrm{Q}} \leq Q_{2}(v, \theta) \leq \underset{\max 2}{Q} \\
& \underset{\min 1}{v} \leq v_{1} \leq \underset{\max 1}{v} \\
& \underset{\min 2}{v} \leq v_{2} \leq \underset{\operatorname{ma}}{v} \\
& \underset{\min 3}{v} \leq v_{3} \leq \underset{\max 3}{v}
\end{aligned}
$$

em que:

$$
\begin{aligned}
& \left.f(v, \theta)=4\left[v_{1}^{2}+v_{3}^{2}-2 v_{1} v_{3} \cos \theta_{13}\right)+\left(v_{2}^{2}+v_{3}^{2}-2 v_{2} v_{3} \cos \theta_{23}\right)\right] \\
& \Delta P_{2}=4 v_{2}^{2}-4 v_{2} v_{3} \cos \theta_{23}+10 v_{2} v_{3} \operatorname{sen} \theta_{23}-1,7 \\
& \Delta P_{3}=\left(4 v_{3}^{2}-4 v_{3} v_{2} \cos \theta_{32}+10 v_{3} v_{2} \operatorname{sen} \theta_{32}\right)+\left(4 v_{3}^{2}-4 v_{3} v_{1} \cos \theta_{31}+5 v_{3} v_{1} \operatorname{sen} \theta_{31}\right)+2 \\
& \Delta Q_{3}=\left(10 v_{3}^{2}-10 v_{3} v_{2} \cos \theta_{32}-4 v_{3} v_{2} \operatorname{sen} \theta_{32}\right)+\left(5 v_{3}^{2}-5 v_{3} v_{1} \cos \theta_{31}-4 v_{3} v_{1} \operatorname{sen} \theta_{31}\right)+1 \\
& Q_{2}(v, \theta)=v_{2}\left(10 v_{2}-10 v_{3} \cos \theta_{23}-4 v_{3} \operatorname{sen} \theta_{23}\right.
\end{aligned}
$$

A Tabela 1 apresenta o estado inicial do sistema e os limites para as restrições de tensão e reativo.

Tabela1: Estado Inicial e limites para tensões e reativos para o sistema 3 barras.

\begin{tabular}{|c|c|c|c|c|}
\hline $\begin{array}{c}\text { Barra } \\
\mathbf{k}\end{array}$ & $\begin{array}{c}\mathbf{V}_{\mathbf{k}} \\
(\mathbf{p . u})\end{array}$ & $\begin{array}{c}\text { Ang k } \\
(\mathbf{r a d})\end{array}$ & $\begin{array}{c}\mathbf{V}_{\min \mathbf{k}} \\
(\mathbf{p . u})\end{array}$ & $\begin{array}{c}\mathbf{V}_{\operatorname{max~k}} \\
(\mathbf{p . u})\end{array}$ \\
\hline 1 & 1 & 0 & 0,80 & 1,20 \\
\hline 2 & 1 & 0 & 0,80 & 1,20 \\
\hline 3 & 1 & 0 & 0,99 & 1,01 \\
\hline
\end{tabular}

Os valores iniciais atribuídos para o parâmetro de Barreira $\mu=0,001$ e os multiplicadores de Lagrange para restrições de igualdade $\lambda=0$ e os multiplicadores de 
Lagrange para restrições de desigualdade $\pi=0,05$.

A tabela 2 apresenta os valores das tensões e ângulos na solução do sistema.

Tabela 2: Variáveis do Sistema de 3 barras no ponto de operação ótimo.

\begin{tabular}{|c|c|c|}
\hline Barra $_{\mathbf{k}}$ & $\mathbf{V}_{\mathbf{k}}$ & Ang $_{\mathbf{K}}$ \\
\hline 1 & 1,08 & 0 \\
\hline 2 & 1,13 & 0,0755 \\
\hline 3 & 1,01 & $-0,0224$ \\
\hline
\end{tabular}

Na tabela 3 encontra-se um resumo do processo de otimização para os dois métodos separadamente.

Tabela 3: O processo de otimização do sistema de 3 barras para os dois métodos.

\begin{tabular}{|r|r|r|r|r|r|r|r|}
\hline \multicolumn{4}{|c|}{ Método Barreira Logarítmica } & \multicolumn{4}{|c|}{ Método Barreira Modificada } \\
\hline Iteração & $\begin{array}{l}\text { Perdas } \\
(\mathbf{M W})\end{array}$ & $\begin{array}{l}\text { Passo } \\
\text { Primal }\end{array}$ & $\begin{array}{l}\text { Passo } \\
\text { Dual }\end{array}$ & $\begin{array}{l}\text { Perdas } \\
\text { Iteração } \\
\text { (MW) }\end{array}$ & $\begin{array}{l}\text { Passo } \\
\text { Primal }\end{array}$ & $\begin{array}{l}\text { Passo } \\
\text { Dual }\end{array}$ \\
\hline 1 & 9,39 & 0,823 & 0,6121 & 1 & 9,95 & 0,853 & 0,9489 \\
\hline 2 & 13,24 & 0,9995 & 0,12 & 2 & 13,18 & 0,9995 & 0,012 \\
\hline 3 & 13,16 & 0,9995 & 0,00006 & 3 & 12,7 & 0,0529 & 0,9172 \\
\hline 4 & 12,65 & 0,3318 & 0,00003 & 4 & 12,7 & 0,0001 & 0,9995 \\
\hline 5 & 12,65 & 0,0437 & 0,9995 & 5 & 12,67 & 0,8761 & 0,9995 \\
\hline 6 & 12,67 & 0,9995 & 0,9995 & 6 & 12,67 & 0,9993 & 0,9995 \\
\hline 7 & 12,67 & 0,8019 & 0,9995 & & & & \\
\hline
\end{tabular}

Na tabela 4 são apresentados os resultados obtidos através da mistura dos métodos.

Tabela4: Mistura de Métodos

\begin{tabular}{|r|r|r|r|}
\hline Iteração & $\begin{array}{l}\text { Perdas } \\
\text { (MW) }\end{array}$ & $\begin{array}{l}\text { Passo } \\
\text { Primal }\end{array}$ & $\begin{array}{l}\text { Passo } \\
\text { Dual }\end{array}$ \\
\hline \multicolumn{4}{|c|}{ Método Barreira Logarítmica } \\
\hline 1 & 9,39 & 0,824 & 0,6122 \\
\hline 2 & 13,24 & 0,9995 & 0,12 \\
\hline 3 & 13,16 & 0,9995 & 0,00006 \\
\hline \multicolumn{4}{|c|}{ Método Barreira Modificada } \\
\hline 4 & 12,65 & 0,0378 & 0,3296 \\
\hline 5 & 12,65 & 0,0007 & 0,9995 \\
\hline 6 & 12,66 & 0,658 & 0,9995 \\
\hline
\end{tabular}


O ponto de operação obtido atingiu a mesma solução do método proposto por DOMMEL e TINNEY [9], com uma precisão de $10^{-3}$ p.u. para as equações do fluxo de potência. Na solução do problema, todas as restrições de igualdade e desigualdade e as condições de KKT estão satisfeitas.

\section{CONCLUSÃO}

Neste trabalho apresentou-se uma abordagem denominada de mistura da Função Barreira e da Função Barreira Modificada para a resolução do problema de Fluxo de Potência Ótimo Reativo. Os resultados dos testes com o sistema 3 barras mostram a aplicabilidade da abordagem proposta. O método pode ser aplicado a outros problemas da área de sistemas elétricos de potência como, por exemplo, colapso de tensão, máximo carregamento, entre outros.

\section{AGRADECIMENTOS}

Os autores agradecem a Coordenação de Aperfeiçoamento de Pessoal de Nível Superior (CAPES) e ao Conselho Nacional de Desenvolvimento Científico e Tecnológico (Bolsista - CNPq) pelo apoio financeiro.

\section{REFERÊNCIA}

[1] BAPTISTA, E. C.; BELATI, E. A.; COSTA, G. R. M. Um Método Primal-Dual para Resolução do Problema de Fluxo de Potência Ótimo. Pesquisa Operacional,v. 24,n. 2, p. 215 226, 2004

[2] BAPTISTA, E. C.; BELATI, E. A.; COSTA, G. R. M. Logarithmic Barrier-Augmented Lagrangian Function to the Optimal Power Flow Problem. International Journal of Electrical Power \& Energy Systems, v. 27,n. 7,p. 528-532, 2005.

[3] BAPtistA, E. C.; BELATI, E. A.; SOUSA, V. A.; COSTA, G. R. M. Primal-Dual Logarithmic Barrier and Augmented Lagrangian Function to the Loss Minimization in Power Systems. Electric power components and systems,v. 34,n. 7,p. 1-6, 2006 a.

[4] BAPTISTA, E. C.; SOUSA, V. A.; COSTA, G. R. M. A Função Barreira Modificada e o Problema de Fluxo de Potência Ótimo. Tema, v.7,n.1,p.21-30, 2006b

[5] CAPITANESCU, F.;GLAVIC, M.;ERNEST,D.;WEHENKEL,L.Interior point based algorithms for the solution of optimal power flow problems. Electric Power Systems Research,77,p.508-517,2007.

[6]CAPITANESCU,F.;RAMOS,J.L.M.;PANCIATICI,P;KIRSCHEN,D.;MARCOLINI,A.M.; PLATBROOD,L.;WEHEKEL,L. State-of-the-art,challengs, and future trends in security constrained optimal power flow. Electric Power Systems Research, n. 81, p.1731-17411. 2011.

[7] CAPITANESCU, F.; WEHENKEL,L. Experiments with the interior point method for solving large scale optimal power flow problems. Electric Power Systems Research, 95,p.276283.2013. 
[8] CARROL, C.W. The Created Response Surface Technique for Optimizing Nonlinear Restrained Systems. Operations Research,v. 9,p.169-184, 1961

[9] DOMMEL, H. W. and TINNEY, W. F. (1968). Optimal Power Flow Solutions. IEEE Transactions on Power Apparatus and Systems, Vol. 87, No. 10, pp. 1866-1876

[10]FIACCO, A.V.; McCORMICK, G.P. Nonlinear Programming - Sequential Unconstrained Minimization Techniques. New York : Wiley, 1968

[11] FRISCH, K. R. The Logarithmic Potential Method of Convex Programming. University Institute of Economics (manuscript). Oslo, Norway, 1955

[12] GRANVILLE, S. Optimal Reactive Dispatch Through Interior Point Methods. IEEE Transactions on Power Systems, v.9, n.1,p.136-146, 1994.

[13] GRIVA, I.A.Numerical experiments with na interior-exterior point method for nonlinear programming. Journal of Computacional Optmization and Aplications, vol.29,p173-195,2004

[14] MONTICELLI, A. and Liu, W.H.E. (1992). Adaptive Movement Penalty Method for Newton Optimal Power Flow. IEEE Transactions on Power Systems, Vol. 7, No. 1, p. 334341.

[15] POLYAK, R.. Modified barrier functions. Mathematical Programming, v.54,n. 2, p.177$222,1992$.

[16] SOUSA, V. A. ; Da Costa . Minimização de Perdas na Transmissão em Sistemas de Potência pelo Método de Newton-Modificado. In: XXII CILAMCE 2001, 2001, Campinas. 22 Irerian Latin-American Congress on Computational Methods in Engineering, 2001.

[17] SOUSA, V.A.; Resolução do Fluxo de Potência Ótimo Reativo via um Método da Função Lagrangiana Barreira Modificada.2006..Escola de Engenharia de São Carlos, Universidade de São Paulo, São Carlos, p. 126, 2006.

[18] SOUSA, V. A. ; BAPTISTA, E.C ; COSTA, G.R.M . Fluxo de potência ótimo reativo via método da função lagrangiana barreira modificada. Controle \& Automação ,v. 19, p. 8392, 2008.

[19] SOUSA, V. A. ; BAPTISTA, E. C. ; Costa, Geraldo R. M da . Loss Minimization by the Predictor-Corrector Modified Barrier Approach. Electric Power Systems Research, v. 79, p. 803-808, 2009.

[20] SOUSA, V.A. ; Baptista, E.C. ; da Costa, G.R.M. . Optimal reactive power flow via the modified barrier Lagrangian function approach. Electric Power Systems Research (Print), v. 84, p. 159-164, 2012

[21] TORRES, G. L.; QUINTANA, V.H.; TORRES, G.L. Optimal Power Flow in Rectangular Form via an Interior Point Method. IEEE Transactions on Power Systems, v.13, n.4, p.1211-1218, 1998

[22] WU, Y. C.; DEBS, A. S.; MARSTEN, R. E. A direct nonlinear Predictor-Corretor Primal-Dual Interior Point Algorithm for Optimal Power Flows. IEEE Transactions on Power 
Systems, v.9,n. 2, p. 876-883, 1994. 